\title{
AN ANALOGY OF HAHN-BANACH SEPARATION THEOREM FOR NEARLY TOPOLOGICAL LINEAR SPACES
}

\author{
Madhu Ram \\ Department of Mathematics, University of Jammu, \\ Jammu-180006, India \\ madhuram0502@gmail.com
}

\begin{abstract}
In this paper, we introduce the notion of nearly topological linear spaces and use it to formulate an alternative definition of the Hahn-Banach separation theorem. We also give an example of a topological linear space to which the result is not valid. It is shown that $\mathbb{R}$ with its ordinary topology is not a nearly topological linear space.
\end{abstract}

Keywords: Hahn-Banach separation theorem, $\alpha$-open sets, $\alpha$-compact sets, Nearly topological linear spaces.

\section{Introduction}

In this paper, all linear spaces are over the field $\mathbb{K} \in\{\mathbb{R}, \mathbb{C}\}$ unless clear from the context. When we treat $\mathbb{K}$ as a topological space, we mean $\mathbb{K}$ is equipped with its standard topology. For any undefined concepts and terminologies, refer to [9].

Topological linear spaces are intensively studied since they are useful for instance in functional analysis, fixed point theory, equilibrium problems and many others. In functional analysis and fixed point theory, there are many popular theorems which are proven for topological linear spaces like Schauder-Tychonoff fixed point theorem, Hahn Banach separation theorem, etc (for example, see $[1,9,10])$. This paper acquires its inspiration from the following result which is very popular in Functional Analysis and other related branches of Science (see [1, 5, 9, 10], for example) and some papers of its applications (see [2-5, 8], for example):

Theorem 1 (Hahn-Banach Separation Theorem). Let $\mathfrak{a}, \mathfrak{b}$ be disjoint, non-empty convex sets in a topological linear space $L$.

(a) If $\mathfrak{a}$ is open, then there exist a continuous linear functional $\varphi: L \rightarrow \mathbb{K}$ and $\lambda \in \mathbb{R}$ such that $\operatorname{Re} \varphi(x)<\lambda \leq \operatorname{Re} \varphi(y)$, for all $x \in \mathfrak{a}, y \in \mathfrak{b}$.

(b) If $\mathfrak{a}$ is compact, $\mathfrak{b}$ is closed, and $L$ is locally convex, then there exist a continuous linear functional $\varphi: L \rightarrow \mathbb{K}$ and $\alpha, \beta \in \mathbb{R}$ such that $\operatorname{Re} \varphi(x)<\alpha<\beta<\operatorname{Re} \varphi(y)$, for all $x \in \mathfrak{a}$, $y \in \mathfrak{b}$.

A natural question to ask is: Is Theorem 1 still valid if $L$ is not a topological linear space? We exhibit that there is a partial answer to this question for a different class of topological linear spaces.

Definition 1. Let $L$ be a linear space and $\mathfrak{a}$ a subset of $L$. Then $\mathfrak{a}$ is called

(1) convex if $\forall x, y \in \mathfrak{a}$, and $\forall \alpha, \beta \geq 0$ such that $\alpha+\beta=1$, $\alpha x+\beta y \in \mathfrak{a}$; 
(2) absorbing if for each $x \in L, \exists r>0$ such that $\forall \lambda \in \mathbb{K}$ with $|\lambda| \leq r$, we have $\lambda x \in \mathfrak{a}$;

(3) balanced if $\forall x \in \mathfrak{a}$, and $\forall \lambda \in \mathbb{K}$ with $|\lambda| \leq 1$, we have $\lambda x \in \mathfrak{a}$.

Definition 2. Let $L$ be a linear space and $\mathfrak{c}$ be a non-empty subset of $L$ which is absorbing. The Minkowski (or gauge) functional of $\mathfrak{c}$ is a function, $p: L \rightarrow \mathbb{R}$, defined as

$$
p(x)=\inf \{\lambda>0: x \in \lambda \mathfrak{c}\} .
$$

Lemma 1 [9, Theorem 1.35]. Suppose $\mathfrak{c}$ is a convex absorbing set in a vector space L. Then

(1) $p(x+y) \leq p(x)+p(y)$;

(2) $p(\lambda x)=\lambda p(x)$ if $\lambda \geq 0$;

(3) $p$ is a semi-norm if $\mathfrak{c}$ is balanced.

A subset $\mathfrak{a}$ of a topological space $X$ is called $\alpha$-open [7] if $\mathfrak{a} \subseteq \operatorname{Int}(\mathrm{Cl}(\operatorname{Int}(\mathfrak{a})))$. The complement of an $\alpha$-open set is called $\alpha$-closed set. The class of $\alpha$-open sets of a given topological space $X$ forms a topology on $X$ and it is denoted by $\Im^{\alpha}$. In the following, for given a topological space $X$ we write the corresponding topological space $\left(X, \Im^{\alpha}\right)$ by $X^{\alpha}$. A subset $\mathfrak{a}$ of $X$ is called $\alpha$-compact [6] if every cover of $\mathfrak{a}$ by $\alpha$-open sets of $X$ has a finite subcover.

Note that every open set in a topological space is $\alpha$-open, every closed set in a topological space is $\alpha$-closed, and every $\alpha$-compact set in a topological space is compact, but the converse of these implications is not true in general.

Example 1. Consider the topological space $(X, \Im)$ where $X=\mathbb{R}$, and $\Im$ is the usual topology on $\mathbb{R}$. Let

$$
\mathfrak{a}=\left\{x \in \mathbb{R}:-1<x<1, x \neq \frac{1}{n}, n \in \mathbb{N}\right\},
$$

where $\mathbb{N}$ denotes the set of positive integers. Then $\mathfrak{a}$ is $\alpha$-open set in $\mathbb{R}$ which is not open. Further let

$$
\mathfrak{b}=\left\{\frac{1}{n}: n \in \mathbb{N}\right\}
$$

then $\mathfrak{b}$ is not closed set in $\mathbb{R}$ but it is $\alpha$-closed set.

\section{The main results}

Definition 3. We call a pair $(L, \Im)$ (or simply, $L$ if no confusion arises) nearly topological linear space if:

- L is a linear space;

- $\Im$ is a topology on L, with

(1) for each $\alpha$-open set $W$ of $L$ containing the vector sum $x+y$ with $x, y \in L$, there exist $\alpha$-open sets $U$ and $V$ of $L$ containing $x$ and $y$, respectively such that $U+V \subseteq W$, and

(2) for each $\alpha$-open set $W$ of $L$ containing the scalar product $\lambda x$ with $x \in L$ and $\lambda \in \mathbb{K}$, there exist an open set $U$ of $\mathbb{K}$ containing $\lambda$ and an $\alpha$-open set $V$ of $L$ containing $x$ such that $U V \subseteq W$. 
From this definition, we have immediately:

Remark 1. A nearly topological linear space is not necessarily a topological linear space. Conversely, $\mathbb{R}$ with its usual topology is a topological linear space which is not a nearly topological linear space because for $\alpha$-open set

$$
W=\left\{x \in \mathbb{R}:-1<x<1, x \neq \frac{1}{n}, x \neq-\frac{1}{m}, m, n \in \mathbb{N}\right\}
$$

which visibly contains $0=0.0$, there do not exist any open set $U$ in $\mathbb{K}$ containing 0 and $\alpha$-open set $V$ in $L$ containing 0 such that $U V \subseteq W$.

For a nearly topological linear space $L$, consider the mappings,

$$
\begin{gathered}
\sigma_{x}: L^{\alpha} \rightarrow L^{\alpha} \quad \text { defined as } \sigma_{x}(y)=x+y, \\
\pi_{\lambda}: L^{\alpha} \rightarrow L^{\alpha} \quad \text { defined as } \pi_{\lambda}(x)=\lambda x ; \quad x, y \in L, \quad \lambda \in \mathbb{K} .
\end{gathered}
$$

Theorem 2. For a nearly topological linear space $L, \sigma_{x}$ and $\pi_{\lambda}$ are continuous.

P r o o f. Follows from Definition 3.

For a nearly topological linear space $L$, we denote by $Z_{0}$ the class of $\alpha$-open sets of $L$ containing the zero vector of $L$.

Theorem 3. In a nearly topological linear space $L$, the following statements are valid:

(a) Every $\mathfrak{c} \in Z_{0}$ is absorbing and balanced.

(b) If in addition, $\mathfrak{c} \in Z_{0}$ is convex, then the Minkowski functional $p$ of $\mathfrak{c}$ is a semi-norm and the set

$$
\{x \in L: p(x)<1\}=\mathfrak{c} .
$$

P r o o f. (a) Since $0=0.0$, there exist an open set $\mathfrak{u}$ in $\mathbb{K}$ containing 0 , and $\mathfrak{v} \in Z_{0}$ such that $\mathfrak{u v} \subseteq \mathfrak{c}$. Then there exist a real $\epsilon>0$ and an open disk $\mathbb{D}_{\epsilon}$ with center 0 and radius $\epsilon$ such that $\mathbb{D}_{\epsilon} \mathfrak{v} \subseteq \mathfrak{c}$. By Theorem 2, $\pi_{1 / \lambda}$ is continuous, so the set $\mathfrak{a}=\mathbb{D}_{\epsilon} \mathfrak{v} \in Z_{0}$. Clearly, $\mathfrak{a}$ is balanced. Next, by Definition 3, we have that for any element $x \in L$, there exists an open set $\mathfrak{u}$ in $\mathbb{K}$ containing 0 s.t. $\mathfrak{u} x \subseteq \mathfrak{c}$. Then there exist a real $r>0$ and an open disk $\mathbb{D}_{r}$ with center 0 and radius $r$ such that $\mathbb{D}_{r} x \subseteq \mathfrak{c}$, showing that $\mathfrak{c}$ is absorbing.

(b) Follows from Lemma 1.

Theorem 4. Suppose $\mathfrak{a}, \mathfrak{b}$ are disjoint sets in a nearly topological linear space L. If $\mathfrak{a}$ is an $\alpha$-compact set in $L, \mathfrak{b}$ is an $\alpha$-closed set in $L$, then there exists a symmetric set $\mathfrak{u} \in Z_{0}$ such that $(\mathfrak{a}+\mathfrak{u}) \cap(\mathfrak{b}+\mathfrak{u})=\emptyset$.

P r o o f. Let $x \in \mathfrak{a}$ be an element. By Definition 3 , there are $\mathfrak{u}_{1}, \mathfrak{u}_{2} \in Z_{0}$ such that

$$
\left(x+\mathfrak{u}_{1}+\mathfrak{u}_{2}\right) \cap \mathfrak{b}=\emptyset .
$$

Consider,

$$
\mathfrak{u}=\mathfrak{u}_{1} \cap \mathfrak{u}_{2} \cap\left(-\mathfrak{u}_{1}\right) \cap\left(-\mathfrak{u}_{2}\right) .
$$

Since $\pi_{\lambda}$ is continuous, $\mathfrak{u} \in Z_{0}$. Consequently, there is a symmetric set $\mathfrak{u}_{x} \in Z_{0}$ such that

$$
\left(x+\mathfrak{u}_{x}+\mathfrak{u}_{x}+\mathfrak{u}_{x}\right) \cap \mathfrak{b}=\emptyset \Rightarrow\left(x+\mathfrak{u}_{x}+\mathfrak{u}_{x}\right) \cap\left(\mathfrak{b}+\mathfrak{u}_{x}\right)=\emptyset .
$$


In a similar vein, we obtain a family

$$
\mho=\left\{x+\mathfrak{u}_{x}: x \in \mathfrak{a}\right\}
$$

of sets. By Theorem 2, $x+\mathfrak{u}_{x}$ is $\alpha$-open set in $L$. Therefore, for some positive integer $n$, we have

$$
\mathfrak{a} \subseteq \bigcup_{i=1}^{n}\left(x_{i}+\mathfrak{u}_{x_{i}}\right), \quad x_{i} \in \mathfrak{a} \quad \text { for all } i=1,2, \ldots, n .
$$

Let

$$
\nu=\bigcap_{i=1}^{n} \mathfrak{u}_{x_{i}}
$$

Then $\nu \in Z_{0}, \nu=-\nu$, and

$$
(\mathfrak{a}+\nu) \cap(\mathfrak{b}+\nu)=\emptyset
$$

also.

Theorem 5. Suppose $\mathfrak{a}, \mathfrak{b}$ are disjoint, non-empty convex sets in a nearly topological linear space $L$.

(a) If $\mathfrak{a}$ is $\alpha$-open, then there is a linear continuous map $\varphi: L^{\alpha} \rightarrow \mathbb{K}$ such that $\operatorname{Re} \varphi(x)<\operatorname{Re} \varphi(y)$, for every $x \in \mathfrak{a}$ and for every $y \in \mathfrak{b}$.

(b) If $\mathfrak{a}$ is $\alpha$-compact, $\mathfrak{b}$ is $\alpha$-closed and for every $\mathfrak{c} \in Z_{0}$, there exists a convex set $\mathfrak{c}_{0} \in Z_{0}$ such that $\mathfrak{c}_{0} \subseteq \mathfrak{c}$, then there exist a linear continuous map $\varphi: L^{\alpha} \rightarrow \mathbb{K}, \lambda \in \mathbb{R}$ and an $\epsilon>0$ such that $\operatorname{Re} \varphi(x)<\lambda<\lambda+\epsilon<\operatorname{Re} \varphi(y)$, for every $x \in \mathfrak{a}$ and for every $y \in \mathfrak{b}$.

P r o o f. (a) We have two cases.

Case I: $\mathbb{K}=\mathbb{R}$. Fix $x_{0} \in \mathfrak{a}, y_{0} \in \mathfrak{b}$. Let

$$
\mathfrak{c}=\mathfrak{a}-\mathfrak{b}+y_{0}-x_{0} .
$$

Then $\mathfrak{c}$ is convex set in $L$, with $\mathfrak{c} \in Z_{0}$. Let $p$ be the Minkowski functional of $\mathfrak{c}$. By Theorem $3, p$ is semi-norm on $L$. Since

$$
\mathfrak{a} \cap \mathfrak{b}=\emptyset, \quad y_{0}-x_{0}=w \notin \mathfrak{c}
$$

and so $p(w) \geq 1$.

Consider the linear subspace $M=\mathbb{R} w$ of $L$ and define $\psi: M \rightarrow \mathbb{R}$ by $\psi(t w)=t$. Evidently, $\psi$ is a linear functional on $M$ s.t.

$$
\psi(y) \leq p(y), \quad \forall y \in M .
$$

By Hahn-Banach extension theorem, there is a linear functional $\varphi$ on $L$ s.t.

$$
\left.\varphi\right|_{M}=\psi \quad \text { and } \quad \varphi(y) \leq p(y), \quad \forall y \in L .
$$

Now, for sufficiently small $\epsilon>0$, take $\mathfrak{u}=(\epsilon \mathfrak{c}) \cap(-\epsilon \mathfrak{c})$. By Theorem $2, \mathfrak{u} \in Z_{0}$, and for every $x \in \mathfrak{u}$, $\pm x \in \epsilon \mathfrak{c}$, giving us $\epsilon^{-1}( \pm x) \in \mathfrak{c}$. By Theorem 3, $p( \pm x)<\epsilon$. That is, $|\varphi(x)|<\epsilon$ for all $x \in \mathfrak{u}$.

Next, since for every $x \in \mathfrak{a}, y \in \mathfrak{b}, \varphi(x-y)<0$ so we have

$$
\varphi(x) \leq \lambda \leq \varphi(y), \quad \text { forall } \quad x \in \mathfrak{a}, \quad y \in \mathfrak{b},
$$

where $\lambda=\sup \{\varphi(x): x \in \mathfrak{a}\}$.

Suppose there exists some $a_{0} \in \mathfrak{a}$ s.t. $\varphi\left(a_{0}\right)=\lambda$. By the continuity of the map

$$
\mathbb{R} \ni \alpha \mapsto a_{0}+\alpha w \in L^{\alpha}
$$


we have a real number $\epsilon>0$ such that

$$
a_{0}+\alpha w \in \mathfrak{a}, \quad \text { for each } \quad \alpha \in \mathbb{R} \text { satisfying }|\alpha| \leq \epsilon .
$$

In particular, $a_{0}+\epsilon w \in \mathfrak{a}$, showing that $\lambda+\epsilon \leq \lambda$, which is impossible.

Case II: $\mathbb{K}=\mathbb{C}$. The above case gives us a linear continuous function $\varphi: L^{\alpha} \rightarrow \mathbb{R}$ with the requisite properties. Then considering the function $\psi(\varsigma)=\varphi(\varsigma)-i \varphi(i \varsigma)$ is the required function, where $i=\sqrt{-1}$.

(b) By Theorem 4, there exists a set $\mathfrak{u} \in Z_{0}$ such that $(\mathfrak{a}+\mathfrak{u}) \cap \mathfrak{b}=\emptyset$. Then part (a) indicates that there are a continuous linear function $\varphi: L^{\alpha} \rightarrow \mathbb{K}$, and $\lambda \in \mathbb{R}$ such that

$$
\operatorname{Re} \varphi(x)<\lambda \leq \operatorname{Re} \varphi(y), \quad \text { for every } \quad x \in \mathfrak{a}+\mathfrak{u}, \quad y \in \mathfrak{b} .
$$

Since $\mathfrak{a}$ is compact proper subset of $\mathfrak{a}+\mathfrak{u} \subseteq L^{\alpha}, \operatorname{Re} \varphi(\mathfrak{a})$ is compact proper subset of $\operatorname{Re} \varphi(\mathfrak{a}+\mathfrak{u})$. Thus, there exists $\lambda>0$ such that

$$
\operatorname{Re} \varphi(x)<\lambda<\lambda+\epsilon<\operatorname{Re} \varphi(y), \quad \text { for every } \quad x \in \mathfrak{a} \quad \text { and for every } \quad y \in \mathfrak{b}
$$

Whence the proof easily follows.

Corollary 1. Suppose $\mathfrak{b}$ is a convex, balanced, $\alpha$-closed set in a nearly topological linear space $L$. If $x_{0} \in L$, but $x_{0} \notin \mathfrak{b}$ and for every $\mathfrak{v} \in Z_{0}$, there exists a convex set $\mathfrak{u} \in Z_{0}$ such that $\mathfrak{v} \subseteq \mathfrak{u}$, then there is a continuous linear functional $\varphi: L^{\alpha} \rightarrow \mathbb{K}$ such that

$$
|\varphi(x)| \leq 1, \quad \text { for all } \quad x \in \mathfrak{b}, \quad \text { and } \quad\left|\varphi\left(x_{0}\right)\right|>1
$$

\section{Conclusion}

In this paper, we introduced the notion of nearly topological linear spaces and formulated an alternative definition of Hahn-Banach separation theorem by using the notion of $\alpha$-open sets in topological spaces in the sense of Njastad. It is shown that $\mathbb{R}$ with its ordinary topology is not a nearly topological linear space.

If we endow $\mathbb{C}$, the real linear space of complex numbers with the topology generated by the family of sets of the form

$$
D_{r, \epsilon}=\left\{x+i y: x, y \in \mathbb{R}, r-\epsilon<x<r+\epsilon, i^{2}=-1\right\}
$$

with $r \in \mathbb{R}$ and $\epsilon>0$, then $\mathbb{C}$ is a nearly topological linear space.

Besides checking the validity of results of topological linear spaces in the field of nearly topological linear spaces, it will be a good contribution finding some more examples of nearly topological linear spaces which satisfy some separation axioms and Theorem 5.

\section{Acknowledgements}

The author thanks the referee and the reviewers for their valuable comments/suggestions which improved the quality of the paper. 


\section{REFERENCES}

1. Amar A. B., Cherif M. A., Mnif M. Fixed-point theory on a Frechet topological vector space. Int. J. Math. Math. Sci., 2011. Vol. 2011. Art. no. 390720. P. 1-9. DOI: 10.1155/2011/390720

2. Deutsch F. R., Maserick P. H. Applications of the Hahn-Banach theorem in approximation theory. SIAM Rev., 1967. Vol. 9, No. 3. P. 516-530. URL: https://www.jstor.org/stable/2027994

3. Deutsch F., Hundal H., Zikatanov L. Some Applications of the Hahn-Banach Separation Theorem, 2017. 26 p. arXiv:1712.10250v1 [math.FA]

4. Helton J. W., Klep I., McCullough S. The Tracial Hahn-Banach Theorem, Polar Duals, Matrix Convex Sets, and Projections of Free Spectrahedra, 2014. 56 p. arXiv:1407.8198 [math.OA]

5. Luna-Elizarrarás M. E., Perez-Regalado C. O., Shapiro M. On linear functionals and Hahn-Banach theorems for hyperbolic and bicomplex modules. Adv. Appl. Clifford Algebr., 2014. Vol. 24. P. 1105-1129. DOI: $10.1007 / \mathrm{s} 00006-014-0503-\mathrm{z}$

6. Maheshwari S. N., Thakur S. S. On $\alpha$-compact spaces. Bull. Inst. Math. Acad. Sin. (N.S.), 1985. Vol. 13, No. 4. P. 341-347.

7. Njåstad O. On some classes of nearly open sets. Pacific J. Math., 1965. Vol. 15, No. 3. P. 961-970.

8. Nörtemann S. The Hahn-Banach theorem for partially ordered totally convex, positively convex and superconvex modules. Appl. Categ. Structures, 2002. Vol. 10, p. 417-429. DOI: 10.1023/A:1016390813177

9. Rudin W. Functional Analysis. 2nd ed. Singapore: McGraw-Hill Inc., 1991. 448 p.

10. Schaefer H.H. Topological Vector Spaces. New York: Springer-Verlag, 1971. 296 p. DOI: $10.1007 / 978-1-4684-9928-5$ 